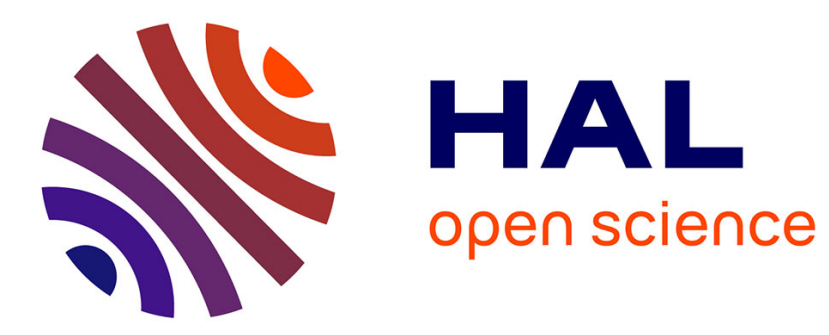

\title{
Transcriptional integration of the plant responses to iron availability
}

Fei Gao, Christian Dubos

\section{To cite this version:}

Fei Gao, Christian Dubos. Transcriptional integration of the plant responses to iron availability. Journal of Experimental Botany, 2020, 2056-2070 (6), pp.2056-2070. 10.1093/jxb/eraa556 . hal03031725

\section{HAL Id: hal-03031725 \\ https://hal.inrae.fr/hal-03031725}

Submitted on 30 Nov 2020

HAL is a multi-disciplinary open access archive for the deposit and dissemination of scientific research documents, whether they are published or not. The documents may come from teaching and research institutions in France or abroad, or from public or private research centers.
L'archive ouverte pluridisciplinaire HAL, est destinée au dépôt et à la diffusion de documents scientifiques de niveau recherche, publiés ou non, émanant des établissements d'enseignement et de recherche français ou étrangers, des laboratoires publics ou privés.

\section{(ㅇ)(1) $\$$}

Distributed under a Creative Commons Attribution - NonCommercial - NoDerivatives| 4.0 


\section{Transcriptional integration of the plant responses to iron availability}

Fei Gao ${ }^{1} \&$ Christian Dubos ${ }^{1, *}$

${ }^{1}$ BPMP, Univ Montpellier, CNRS, INRAE, Institut Agro, Montpellier, France

* Corresponding author (Tel: 0033 (0)499 6128 18)

E-mails: Fei Gao, fei.gao@supagro.fr; Christian Dubos, christian.dubos@inrae.fr

Highlights: Within this review, current knowledge on the control of iron homeostasis in plants is presented and future prospects to improve our understanding of this complex mechanism are provided.

(C) The Author(s) 2020. Published by Oxford University Press on behalf of the Society for Experimental Biology. All rights reserved. For permissions, please email: journals.permissions@oup.com 


\begin{abstract}
Iron is one of the most important micronutrient for plant growth and development. It functions as the enzyme cofactor or component of electron transport chains in various vital metabolic processes, including photosynthesis, respiration and amino acid biosynthesis. For maintaining iron homeostasis, and therefore preventing any deficiency or excess that could be detrimental, plants have evolved complex transcriptional regulatory networks to tightly control iron uptake, translocation, assimilation and storage. Such regulatory networks are composed of various transcription factors among them members from the basic helix-loophelix (bHLH) family play an essential role. Here, we first review recent advances in understanding the roles of bHLH transcription factors involved in the regulatory cascade controlling iron homeostasis in the model plant Arabidopsis, extending it to rice and other plant species. The importance of other classes of transcription factors will also be discussed. Secondly, we elaborate on the posttranslational mechanisms involyed in the regulation of these regulatory networks. Finally, we give some perspectives on future research that should be conducted in order to precisely depict how plants control the homeostasis of this micronutrient.
\end{abstract}

Key words: Arabidopsis thaliana, basic helix-loop-helix, bHLH, iron homeostasis, rice, transcription factor. 


\section{Introduction}

Iron $(\mathrm{Fe})$ is an essential micronutrient for almost all living organisms. In humans, iron deficiency anemia is a major global health issue affecting about one billion people worldwide (Camaschella, 2015). Therefore, increasing the iron content in plants, especially in crops (biofortification), would have enormous benefits for human health. To achieve this goal, a critical question is to uncover the mechanisms controlling iron homeostasis in plants. Like in humans, iron is an essential microelement for plant growth and development. It functions in various vital metabolic processes (e.g. photosynthesis, respiration, amino acid biosynthesis) by acting as cofactor for several metalloproteins or component of electron transport chains (Hänsch and Mendel, 2009; Touraine et al., 2019). However, the excess of iron is deleterious to plants because of its capacity to interact with oxygen, generating reactive oxygen species (ROS) via the Fenton Reaction. Thus, the levels of iron in plant cells must be tightly regulated to avoid iron deficiency or iron excess, both of which severely affecting crops yield and the quality of their derived products (Briat et al., 2015).

Although iron is the fourth most-abundant element on earth, much of it is not readily available for plant use due to the poor solubility of its main (hydro)oxides, especially in neutral-to-alkaline soils (Guerinot and Yi, 1994; Colombo et al., 2018). To adapt to low iron conditions and acquire iron from soil, higher plants have evolved two different strategies (Marschner and Römheld, 1994) (Figure 1). Dicots and non-graminaceous monocots (nongrass species) employ the reduction strategy (named Strategy I). The graminaceous utilize the chelation strategy (named Strategy II). In strategy I, non-grass plants are able to acidify the rhizosphere to promote iron solubility through protons release and mobilize $\mathrm{Fe}^{3+}$ through coumarins (i.e. fraxetin and sideretin) or riboflavins secretion (Santi and Schmidt, 2009;

Fourcroy et al., 2016; Robe et al., 2020). Following iron mobilization, $\mathrm{Fe}^{3+}$ is reduced into $\mathrm{Fe}^{2+}$ (a more soluble form of iron) that is subsequently transported into the root epidermal cells via high affinity iron transporters from the Zrt/Irt-like protein (ZIP) family.

In Arabidopsis thaliana, the release of protons into the rhizosphere is insured by the $\mathrm{H}^{+}$ATPASE 2 (AHA2; Santi and Schmidt, 2009) whereas the reduction of iron and its translocation into the roots are ensured by FERRIC REDUCTION OXIDASE 2 (FRO2) and IRON-REGULATED TRANSPORTER1 (IRT1), respectively (Brumbarova et al., 2015; Connorton et al., 2017). AHA2, FRO2 and IRT1 associate into a complex at the surface of the 
root epidermal cells most likely allowing optimizing iron acquisition and therefore its uptake (Martín-Barranco et al., 2020). Coumarins secretion is ensure by the PLEIOTROPIC DRUG RESISTANCE 9/ATP-BINDING CASSETTE G37 (PDR9/ABCG37) transporter (Fourcroy et al., 2014; Fourcroy et al., 2016). In strategy II, plants biosynthesize and secrete phytosiderophores of the mugineic acid family (MAs) into the rhizosphere to chelate $\mathrm{Fe}^{3+}$ (Kobayashi et al., 2014). $\mathrm{Fe}^{3+}$-MAs complexes are then transported into root cells by transporters of the YELLOW STRIPE 1 (YS1) and YELLOW STRIPE 1-like (YSL) family (Inoue et al., 2009; Murata et al., 2006). Interestingly, it was recently shown that some plant species might use both strategies. For instance, when grown in waterlogged soil condition, rice (Oryza sativa), a graminaceous species, acquires $\mathrm{Fe}^{2+}$ from the soil through the activity of two $\mathrm{Fe}^{2+}$ transporters, OsIRT1 and OsIRT2 (Ishimaru et al., 2006). The secretion of caffeic and protocatechuic acids via the PHENOLICS EFFLUX ZERO 2 (PEZ2) transporter participates to $\mathrm{Fe}^{3+}$ mobilization and reduction into $\mathrm{Fe}^{2+}$ (Bashir et al., 2011). If iron acquisition is the first step that participates to the maintenance of iron homeostasis in plants, it should be noted that it is not the sole mechanism involved in this process. Iron translocation, compartmentalization, assimilation and storage are also important processes required for the maintenance of iron homeostasis at the cellular and subcellular levels, throughout the whole plant body (Kobayashi and Nishizawa, 2012; Kobayashi et al., 2019).

Gene regulation is a crucial step for coping with iron fluctuations. For instance, FRO2 and IRT1 expression is induced when iron availability is low whereas the expression of the genes encoding the iron storage ferritin proteins (i.e. FER1, 3 and 4) is induced in response to iron excess (Tissot et al., 2019). How plants control iron homeostasis, by regulating the expression of genes involved in the various facets of this complex mechanism, was a critical question for the past three decades. To address this question, several studies mostly based on forward and reverse genetic approaches were conducted, leading to the identification and characterization of several transcription factors (TFs). These studies, mostly conducted in Arabidopsis and rice, allowed establishing regulatory networks controlling iron homeostasis in which basic helix-loop-helix (bHLH) TFs play a preponderant role ( Gao et al., 2019; Gao et al., 2020a; Li et al., 2019) (Figure 2 and Table 1). bHLH proteins form one of the largest families of TFs (Heim et al., 2003) known to modulate several facets of plant growth and development, including cell differentiation, secondary metabolite biosynthesis, hormone signaling or the responses to environmental factors (Carretero-Paulet et al., 2010). Indeed, 
additional TFs and protein regulating TFs activity were also identified (Yan et al., 2016; Palmer et al., 2013; Rodríguez-Celma et al., 2019a).

Within this review, current knowledge on the control of iron homeostasis by TFs, especially from the bHLH family, will be presented and discussed with a special emphasis on the latest findings. The transcriptional and posttranslational regulation of the iron homeostasis regulatory networks will also be documented. Last, some perspectives on future research to be conducted in order to improve our understanding of this complex mechanism will be provided.

\section{The transcriptional regulation of iron homeostasis in strategy I plants, a preponderant role for bHLH TFs}

The model plant Arabidopsis has allowed the identification of several factors involved in the regulation of iron homeostasis, notably by studying its response to iron deficiency. Such studies highlighted that the regulation of iron homeostasis was essentially occurring at the transcriptional level and was involving several TFs, in particular those of the bHLH family. This topic has also been covered in recent reviews by Gao et al., 2019; Kobayashi, 2019; Kobayashi et al., 2019; Wu and Ling, 2019; Schwarz and Bauer, 2020.

To date, at least six bHLH TF subfamilies (Heim et al., 2003), encoding 17 different proteins, are known to participate to the maintenance of iron homeostasis in Arabidopsis. These bHLH TFs form an intricate regulatory network composed of two interconnected regulatory modules (Figure 3).

The first module relies on the activity of FIT/bHLH29 (FER-LIKE IRON DEFICIENCY INDUCED TRANSCRIPTION FACTOR), a clade IIIa bHLH TF (Colangelo and Guerinot, 2004; Jakoby et al., 2004; Yuan et al., 2005). FIT/bHLH29 is a direct regulator of IRT1 and FRO2 expression, highlighting its central role for the regulation of the iron uptake machinery (Wang et al., 2013b). FIT/bHLH29 activity relies on its interaction with the four members of the Ib bHLH clade (i.e. bHLH38, bHLH39, bHLH100 and bHLH101), forming heterodimer complexes displaying partial redundant activities (Colangelo and Guerinot, 2004; Maurer et al., 2014; Yuan et al., 2008; Wang et al., 2013b) (Figure 3). Recently, the members of the IVa bHLH clade (i.e. bHLH18, bHLH19, bHLH20 and bHLH25) were identified as FIT/bHLH29 interacting proteins (Cui et al., 2018). These interactions were shown to 
promote the degradation of FIT/bHLH29 via the $26 \mathrm{~S}$ proteasome pathway, in a jasmonic acid-dependent manner (Cui et al., 2018). It is noteworthy that the Ib bHLH and the IVa bHLH TFs antagonize the activity of each other in regulating FIT/bHLH29 protein accumulation to tightly regulate the iron uptake machinery in response to different environmental stimuli (Cui et al., 2018) (Figure 3).

The second module acts upstream from FIT/bHLH29. It involves the four members of the IVc bHLH clade, namely ILR3/bHLH105 (IAA-LEUCINE RESISTANT 3), IDT1/bHLH34 (IRON DEFICIENCY TOLERANT 1), bHLH104 and bHLH115. These four TFs play additive roles in the iron deficiency responses and their activity is thought to rely, at least in part, on their ability to form homo- or heterodimers (Li et al., 2016; Zhang et al., 2015; Liang et al., 2017) (Figure 3). In response to iron deficiency, these TFs directly activate the expression of clade Ib bHLH and indirectly the one of FIT/bHLH29 (Li et al., 2016; Zhang et al., 2015; Liang et al., 2017).

Clade IVb bHLH TFs (i.e. PYE/bHLH47, bHLH11 and URI/bHLH121), also participate to the regulation of iron homeostasis in Arabidopsis. PYE/bHLH47 is a negative regulator (Long et al., 2010), which contains an EAR motif at its C-terminal region (i.e. DLNxxP; Kagale and Rozwadowski, 2011) that directly represses the expression of genes participating to the maintenance of iron homeostasis. Interaction studies highlighted that PYE/bHLH47 could heterodimerize with ILR3/bHLH105 and bHLH115 (Long et al., 2010; Zhang et al., 2015; Tissot et al., 2019). However, the role of these interactions was still a matter of debate until recently. In a recent study, ILR3/bHLH105 was found to play a central role in the regulation of iron homeostasis where it acts as both transcriptional activator and repressor of the plant responses to iron deficiency and excess, respectively (Kroh and Pilon, 2019; Tissot et al., 2019). In this study, the authors showed that the repressive activity of ILR3/bHLH105 was conferred by its dimerization with PYE/bHLH47 (Figure 3). The authors also highlighted that ILR3-PYE heterodimers might repress the expression of PYE/bHLH47 when iron availability is not limiting via a negative feedback regulatory loop (Figure 3). bHLH11 is another transcriptional repressor also containing an EAR motif (i.e. LxLxL) in its C-terminal domain (Tanabe et al., 2019). Overexpression studies suggest that bHLH11 inhibits the plant tolerance to iron deficiency and the expression of IRTI and FRO2, most probably by indirectly repressing the expression of FIT/bHLH29 (Tanabe et al., 2019) (Figure 3). In contrast to PYE/bHLH47 and bHLH11, URI/ bHLH121 (UPSTREAM REGULATOR OF 
IRT1) has been recently identified and characterized by three different groups as a positive regulator of the plant responses to iron deficiency (Lei et al., 2020; Lockhart, 2020; Gao et al., 2020a; Kim et al., 2019). URI/bHLH121 can form heterodimers with clade IVc bHLH TFs (Lei et al., 2020; Gao et al., 2020a; Kim et al., 2019) (Figure 3). These interactions participate to the relocation of URI/bHLH121 from the cytosol into the nucleus (Lei et al., 2020). It also suggests that the transcriptional activation of clade IVc bHLH target genes requires, at least in part, URI/bHLH121(Lei et al., 2020; Gao et al., 2020a; Kim et al., 2019). In support of this assertion, it was demonstrated that several genes directly targeted by URI/bHLH121 are identical to that of clade IVc bHLH TFs (Figure 3). The expression of FIT/bHLH29 also relies on URI/bHLH121 activity, most probably via an indirect mechanism (Lei et al., 2020; Gao et al., 2020a; Kim et al., 2019). It is noteworthy that under iron deficiency condition, URI/bHLH121 accumulates in its phosphorylated form (Figure 3) that increases its binding capacity to the promoter region of its target genes such as the clade $\mathrm{Ib}$ bHLH TFs (Kim et al., 2019). Interestingly, both URI/bHLH121 transcript and protein accumulate constitutively regardless of iron status (Gao et al., 2020a; Kim et al., 2019). In contrast, URI/bHLH121 cellular localization in roots differs depending on iron availability (Gao et al., 2020a). When iron is not limiting, URI/bHLH121 mainly localizes in the stele and the endodermis, whereas under iron deficiency condition, URI/bHLH121 is primarily observed in the cortex and the epidermis cells, where it promotes iron uptake (Gao et al., 2020a). The thorough characterization of URI/bHLH121 indicates that it plays a key role in the control of plant iron homeostasis mainly because it directly or indirectly regulates the expression of most of the known genes involved in this regulatory network (Gao et al., 2020a; Kim et al., 2019) (Figure 3). Interestingly, it was recently reported that URI/bHLH121 directly activates FER1, FER3 and FER4 expression when iron availability is not in excess, indicating that URI/bHLH121 positively regulates the transient storage of iron in addition to the iron deficiency response (Gao et al., 2020b).

Functional homologs of most of the above-described Arabidopsis bHLH TFs have been characterized in several dicots (Figure 2, Table 1) indicating that this regulatory mechanism is most likely conserved within strategy I plants. 


\section{bHLH TFs involved in the regulation of iron homeostasis in strategy II plants}

As stated above, plants have developed two different strategies to take up iron from the soil, distinguishing the non-graminaceous (Strategy I, reduction strategy) and graminaceous (Strategy II, chelation strategy) species (Figure 1). However, the in depth study of the responses to iron deficiency in rice has highlighted that the regulation of iron homeostasis in strategy II plants involves the activity of several bHLH TFs belonging to the same clades than those identified in strategy I plant species (Figures 2 and 4, Table 1; Kobayashi, 2019; Kobayashi et al., 2019).

OsFIT/OsbHLH156 was recently identified as a positive regulator of the iron deficiency responses (Liang et al., 2020; Wang et al., 2020). Loss-of-function of OsFIT/OsbHLH156 resulted in strong iron deficiency symptoms under upland condition, whereas no iron deficiency symptoms were observed when plants were grown in waterlogged soil (Wang et al., 2020). These results imply that the strategy II iron uptake system was impaired in the Osfit mutant, an hypothesis that is supported by the disruption of the expression of strategy II iron uptake related genes. It includes genes encoding enzyme involved in MAs biosynthesis (e.g. NAS1 and NAS2, NICOTIANAMINE SYNTHASE 1 and 2) or $\mathrm{Fe}^{3+}-\mathrm{MA}$ transport (e.g. OSYSL15, YELLOW STRIPE-LIKE 15) (Figure 1) (Liang et al., 2020; Wang et al., 2020). Interestingly, it was demonstrated that OsFIT/OsbHLH156 regulates the expression of OSIRT1 and therefore participates also to the regulation of strategy I iron uptake mechanism (Liang et al., 2020) (Figure 1). OsFIT/OsbHLH156 interacts with OsIRO2/OsbHLH56, a clade $\mathrm{Ib}$ bHLH, and promotes its nuclear accumulation (Figure 4) (Liang et al., 2020; Wang et al., 2020). OsIRO2/OsbHLH56 was the first characterized bHLH TF involved in the control of iron homeostasis in rice, where it acts as a positive regulator of the iron deficiency response (Ogo et al., 2006; Ogo et al., 2007). Expression analysis indicated that the regulation of OsIRT1 expression by OsFIT/OsbHLH156 might be different to that of Arabidopsis or that it might involve other Ib bHLH TFs than OsIRO2/OsbHLH56.

Three out of the four clade IVc bHLH TFs (i.e. OsPRI1/OsbHLH060, OsPRI2/bHLH058, OsPRI3/OsbHLH059; POSITIVE REGULATOR OF IRON HOMEOSTASIS 1, 2 and 3) have been identified in rice as playing a positive role in the iron deficiency responses (Zhang et al., 2017; Kobayashi et al., 2019; Zhang et al., 2020b). The characterization of loss-offunction mutants suggested that these three TFs directly regulate the expression of 
OsIRO2/OsbHLH56, and indirectly the expression of OsNAS1, OSNAS2, and OsYSL15 via OsIRO2/OsbHLH56 (Figure 4) (Zhang et al., 2020b; Zhang et al., 2017). Among the potential direct targets of OsPRI1/OsbHLH060, OsPRI2/bHLH058 and OsPRI3/OsbHLH059, there is also OsIRO3/OsbHLH63 (Figure 4) (Zhang et al., 2020b; Zhang et al., 2017). OsIRO3/OsbHLH63 is a member of the IVb clade and the functional homolog of PYE/bHLH47 (Zheng et al., 2010). Like PYE/bHLH47, OsIRO3/OsbHLH63 functions as a negative regulator of the iron deficiency responses (Zheng et al., 2010). It is likely that OsIRO3/OsbHLH63 activity might antagonize OsIRO2/OsbHLH56 to tightly regulate iron uptake and avoid iron overload (Zhang et al., 2012; Zhang et al., 2020b; Zhang et al., 2017; Zheng et al., 2010). To date, there is no information on the role of OsbHLH057, the fourth member of the rice clade IVc bHLH. Indeed, based on the information gathered in Arabidopsis, it is likely that OsbHLH057 participates to the control of iron homeostasis in rice. Whether OsbHLH057 exerts a minor role in specific cell types or in specific environmental conditions remains to be determined.

OsbHLH133 functions as a negative regulator of iron translocation from roots to shoots (Wang et al., 2013a). It should be noted that OsbHLH133 is the only one member of the clade VIIIc reported to date as involved in the regulation of iron homeostasis in plants. The other members of this clade in Arabidopsis play a positive role in root hair development but have never been associated with the maintenance of iron homeostasis (Bruex et al., 2012). Whether these bHLHs have a similar and conserved role, as that of OsbHLH133 in the regulation of iron homeostasis in strategy II plants, is to be investigated.

The transcriptional regulation of iron homeostasis is not restricted to the activity of bHLH TFs

TFs from other families are involved in regulatory networks acting for iron homeostasis in both strategy I and strategy II plants (Figures 3 and 4, Table 2).

Several R2R3-MYB TFs from different plant species have been shown to be involved in the regulation of iron deficiency responses. In Arabidopsis, MYB10 and MYB72 are two iron deficiency inducible TFs required for proper iron uptake and whose expression is partially dependent on FIT/bHLH29 and URI/bHLH121 activities (Figure 3) (Gao et al., 2020a; Palmer et al., 2013; Zamioudis et al., 2014). Furthermore, MYB72 has been identified as a 
transcriptional activator of genes associated with the biosynthesis and secretion of ironmobilizing coumarins, highlighting its role in strategy I iron uptake system (Stringlis et al., 2018; Zamioudis et al., 2014) (Figure 1). Recently, it was shown that the Arabidopsis MYB28 and MYB29 are at the interface of the plant sensitivity to ammonium stress and the modulation of iron homeostasis (Coleto et al., 2020). Notably, the ammonium-dependent decrease of MYB28 and MYB29 expression (or the loss-of-function of both genes) leads to defects in iron translocation from roots to shoots and to the induction of the expression of FIT/bHLH29, clade Ib bHLHs, MYB72 as well as IMA1/FEP3 and IMA3/FEP1 in roots. MdMYB58, a close homolog of MYB72, was recently characterized as a positive regulator of iron uptake and translocation in apple (Wang et al., 2018). Further investigation showed that MdMYB58 transcriptional activity is inhibited by its heterodimerization with MdSAT1/MdbHLH18, a IVa clade bHLH TF (Wang et al., 2018), In contrast to the abovementioned MYB TFs, MxMYB1 may function as a negative regulator of iron uptake and storage (Shen et al., 2008).

In Arabidopsis, WRKY46 plays a role in iron translocation between root and shoot by directly regulating the expression of VITL1 (VACUOLAR IRON TRANSPORTER-LIKE 1), a potential iron transporter involved in iron sequestration into the vacuoles (Yan et al., 2016; Gollhofer et al., 2014). HAP5A/NF-YC1 is also involved in iron translocation between root and shoot by regulating the expression of NAS1 (Zhu et al., 2020). ERF4 and ERF72 are two Arabidopsis TFs belonging to AP2/ERF family that have been reported as potential negative regulators of iron deficiency responses by repressing the expression of genes involved in iron uptake such as IRT1 (Liu et al., 2017a; Liu et al., 2017b). Similarly, MbERF4 and MbERF72 from Malus baccata as well as MxERF4 from Malus xiaojinensis act as negative regulators of the iron deficiency responses in these two apple species (Liu et al., 2018; Zhang et al., 2020a). In contrast, ERF95 was recently proposed to promote iron storage in Arabidopsis seeds (Sun et al., 2020). However, how iron distribution is controlled in seeds is not clearly established even if it was recently proposed that B3 TFs, which are involved in the regulation of embryo development and seed maturation, might be good candidates (Roschzttardtz et al., 2020).

IDEF1 and IDEF2 (IRON DEFICIENCY-RESPONSIVE ELEMENT FACTOR 1 and 2) are two rice TFs belonging to two different families, the ABI3/VP1 and NAC, respectively (Figure 4) (Kobayashi et al., 2007; Ogo et al., 2008). These two TFs participate to the regulation of iron homeostasis in rice. IDEF1 is required for the coordinated activation of 
genes related to iron uptake and translocation, including OsIRT1, OsNAS1, OsNAS2 and OsYSL15 (Kobayashi et al., 2009; Kobayashi et al., 2007). In addition, IDEF1 positively regulates the expression of OSIRO2, indicating that IDEF1 functions upstream of OsIRO2 in the iron deficiency regulatory network (Figure 4) (Kobayashi et al., 2009; Kobayashi et al., 2007). Interestingly, it was shown that IDEF1 could bind to iron and zinc atoms and that this capacity was necessary for its activity (Kobayashi et al., 2012). It was therefore proposed that IDEF1 could sense the cellular metal ion balance caused by changes in iron availability, suggesting that IDEF1 could be a cellular iron sensor allowing the tight regulation of the iron deficiency responses (Kobayashi et al., 2012). Similarly to IDEF1, IDEF2 plays a positive role in the plant response to iron deficiency (Ogo et al., 2008).

A family of peptides named IMA/FEP (IRONMAN/FE-UPTAKE-INDUCING PEPTIDE) has been reported to play a positive role in iron deficiency responses in Arabidopsis, by regulating a set of deficiency-inducible genes including Ib bHLH TFs, a function that seems to be conserved across plant species (Grillet et al., 2018; Hirayama et al., 2018). Two recent studies showed that URI/bHLH121 is a direct positive regulator of IMAI/FEP3 and IMA2/FEP2 expression (Gao et al., 2020a; Kim et al., 2019). These results indicate that IMAs/FEPs are implicated in the bHLH-dependent regulatory network regulating iron homeostasis. However, the precise regulatory mechanisms by which IMAs/FEPs act are still unknown.

\section{Post-translational regulation of the iron homeostasis regulatory networks}

Protein-protein interactions as well as post-translational modifications (i.e. ubiquitination, sumoylation, phosphorylation) can significantly affect the regulatory activities of TFs. Such mechanisms were shown to play an important role in the maintenance of iron homeostasis in plants. This topic has also been covered in recent reviews by Kobayashi, 2019; RodríguezCelma et al., 2019a; Schwarz and Bauer, 2020; Spielmann and Vert, 2020; Wu and Ling, 2019.

As described earlier, the transcriptional activity of bHLH TFs involved in the control of iron homeostasis is extensively dependent on in vivo protein-protein interactions, in the form of homo- or heterodimers (Figures 3 and 4, Tables 1 and 2). For instance, FIT/bHLH29 heterodimerization with clade Ib bHLH TFs is required for its transcriptional activity and 
stability, whereas its interaction with clade IVa members promotes its degradation via the 26S proteasome pathway (Cui et al., 2018). bHLH39 nuclear localization also depends on its interaction with FIT/bHLH29 since in the cells lacking FIT/bHLH29, bHLH39 localizes predominantly in the cytoplasm (Trofimov et al., 2019). Similarly, OsFIT/OsbHLH156 facilitates the nuclear localization of OsIRO2/OsbHLH156, the functional homolog of bHLH39 in rice, suggesting that this post-translational regulatory mechanism is conserved within the plant kingdom (Liang et al., 2020; Wang et al., 2020). FIT/bHLH29 activity and/or stability are also modulated by its interaction with several additional protein partners that do not belong to the bHLH family of TFs (Figures 3, Tables 1) (reviewed in Kobayashi, 2019; Schwarz and Bauer, 2020; Spielmann and Vert, 2020; Wu and Ling, 2019).

Several ubiquitin E3 ligases have been identified and characterized as negative regulators of iron uptake to avoid potential iron overload by targeting bHLH TFs for their degradation (for details, see Spielmann and Vert, 2020; Rodríguez-Celma et al., 2019a) (Figures 3 and 4). BTS (BRUTUS), whose expression is induced by iron deficiency in roots, is proposed to be a critical iron sensing E3 ubiquitin ligase in Arabidopsis (Long et al., 2010). BTS interacts with ILR3/bHLH105 and bHLH115 to facilitate their degradation via the $26 \mathrm{~S}$ proteasome pathway, allowing the fine tuning of the expression of downstream iron deficiency response genes (Figure 3) (Selote et al., 2015; Long et al., 2010). Similarly, OsHRZ1 and OsHRZ2 (HAEMERYTHRIN MOTIF-CONTAINING REALLY INTERESTING NEW GENE (RING) AND ZINC-FINGER PROTEIN 1 and 2), two rice ubiquitin E3 ligases displaying strong sequence similarities with BTS, have been reported as potential iron sensors playing a negative role in iron acquisition under iron sufficient conditions (Kobayashi et al., 2013). It was shown that OsHRZ1 could interact with OsPRI1/OsbHLH60, OsPRI2/OsbHLH58 and OsPRI3/OsbHLH59 and mediate their degradation via the 26S proteasome (Figure 4) (Zhang et al., 2020b; Zhang et al., 2017). However, different results were reported in another study only validating the sole interactions between OsHRZ1 and OsHRZ2 with OsPRI1/OsbHLH60 and OsPRI2/OsbHLH58 (Kobayashi et al., 2019). Another in vitro ubiquitination study showed that neither OsPRI1/OsbHLH60, nor OsPRI2/OsbHLH58 or OsPRI3/OsbHLH59 were ubiquitinated by OsHRZ1 or by OsHRZ2 (Kobayashi et al., 2019). These discrepancies may be due to the different methodologies and materials used in these studies. Whether these interactions participate to the ubiquitination and degradation of OsPRI1/OsbHLH60, OsPRI2/OsbHLH58 and OsPRI3/OsbHLH59 remains to be further demonstrated and confirmed. IDEF1, whose activity is necessary for HRZs expression, is also 
degraded via the $26 \mathrm{~S}$ proteasome pathway through a yet uncharacterized mechanism (Zhang et al., 2014). However, IDEF1 degradation is inhibited by its interaction with the IBP1.1 (IDEF1-BINDING PROTEINS 1.1) Bowman-Birk trypsin inhibitor protein (Figure 4) (Zhang et al., 2014). BTSL1 and BTSL2, two Arabidopsis homologs of BTS, function redundantly as negative regulators of the iron deficiency response (Hindt et al., 2017). In addition, BTSL1 and BTSL2 could directly target FIT/bHLH29 and promote its ubiquitination and subsequent degradation via the $26 \mathrm{~S}$ proteasome pathway, thus negatively regulating the expression of iron uptake related genes (Figure 3) (Rodríguez-Celma et al., 2019b; Rodríguez-Celma et al., 2019a). In apples, MdBT1 and MdBT2, two BTB-TAZ proteins, interact with MdbHLH104. MdBT proteins interact as well with MdCUL3 (CULLIN-RING UBIQUITIN LIGASE 3) to form MdBT ${ }^{\text {MdCUL3 }}$ complexes required for MdbHLH104 ubiquitination and degradation via the 26S proteasome pathway (Zhao et al., 2016a). In contrast, MdSIZ1, a SIZ/PIAS-type SUMO E3 ligase, directly sumoylates MdbHLH104, especially under iron deficiency conditions, to enhance its stability (Zhou et al., 2019). Recently, it was shown that the mutation of an alanine into valine within IDT1/bHLH34 enhances both its stability and nuclear localization (Sharma and Yeh, 2020). Since this amino acid is conserved among clade IVc bHLH TFs in both monocots and dicots (Sharma and Yeh, 2020), one might speculate that this amino acid plays an important role in regulating the stability of clade IVc bHLH and thus their degradation via the $26 \mathrm{~S}$ proteasome.

Phosphorylation plays also an important role in determining $\mathrm{TF}$ activity in the iron homeostasis networks (Figure 3). In Arabidopsis, FIT/bHLH29 phosphorylation by the calcium-dependent protein kinase CIPK11 (CBL-INTERACTING PROTEIN KINASE 11) positively regulates its activity by favoring its nuclear accumulation and dimerization with bHLH39 (Gratz et al., 2019). CIPK11 is induced and activated via a CBL1/9-mediated $\mathrm{Ca}^{2+}$ sensing pathway under iron deficiency conditions. CIPK21 could also interact with FIT/bHLH29, indicating that this protein kinase might have a potential role in the regulation of the FIT/bHLH29-dependent iron deficiency responses (Gratz et al., 2019). Recently, the phosphorylation of URI/bHLH121 was also reported as a key mechanism that regulates its activity (Kim et al., 2019). It was shown that the phosphorylated form of URI/bHLH121 only accumulates in response to iron deficiency, whereas the turnover of phosphorylated URI/bHLH121 is dependent on BTS activity (Kim et al., 2019). Under iron deficiency, URI/bHLH121 showed enhanced heterodimerization capability with IVc bHLH TFs and increased binding ability to the promoter of its target genes, indicating that the 
phosphorylation of URI/bHLH121 plays a positive role in the iron deficiency responses (Kim et al., 2019). Nevertheless, since URI/bHLH121 can activate the expression of FER1, FER3 and FER4 in the stele when iron availability is not limiting (Gao et al., 2020b), a growth condition where the phosphorylated form of bHLH121 is degraded (Kim et al., 2019), it is likely that URI/bHLH121 is transcriptionally active independently of its phosphorylation state. Interestingly, as stated earlier, the pattern of accumulation of URI/bHLH121 within the root cells is controlled by an iron-dependent mechanism. URI/bHLH121 is preferentially accumulated in the epidermis and the cortex when plants are grown under iron deficiency and in the stele when grown under iron sufficiency. From these observations, one might hypothesize that URI/bHLH121 cellular distribution, rather than its transcriptional activity, is regulated by its phosphorylation state. However, the precise regulatory mechanism leading to the phosphorylation of URI/bHLH121 remains to be characterized.

\section{Conclusions and future prospects}

In the past two decades, remarkable progresses have been made in decrypting the molecular mechanisms that regulate iron homeostasis in both strategy I and strategy II plants (Figure 1). They highlighted that iron homeostasis in plants is regulated at the transcriptional level and involves several bHLH TFs that function in intricate regulatory networks (Figures 2 to 4).

The function of most of these bHLH TFs (i.e. clades Ib, IIIa, IVb and IVc) is conserved among grass and non-grass species (Figure 2), in contrast to the downstream target genes of iron acquisition machinery, which are distinctive in strategy I and strategy II plants (Figure 1). How these bHLH functional homologs have evolved to target different genes is an intriguing question. bHLH from clade IVa are involved in the regulation of iron homeostasis in Arabidopsis but whether or not this clade of bHLH TFs has a similar role in other plant species needs to be investigated. Additional investigations will also be necessary to determine if the non-grass homologs of OsbHLH133 (clade VIIIc) play a role in the control of iron homeostasis. Other regulatory proteins are also conserved between plants from both strategies. It is for instance the case for MYB and ERF TFs (Table 2) or for haemerythrin E3ubiquitin ligases. Interestingly, BTB-TAZ-CUL3 ubiquitin ligase complexes participate to the degradation of clade IVc bHLH in apples. To date, such mechanism has only been observed in apple, raising the question of the conservation of this regulatory mechanism in other plants species, from non-grass to grass and from perennials to annuals. Ubiquitination, 
as well as phosphorylation and sumoylation, are crucial posttranslational modifications for the regulation of key bHLH TFs activities involved in the iron homeostasis networks, notably clade IVc bHLH FIT/bHLH29 and URI/bHLH121. Whether such modifications participate to the regulation of other TF activities within this network is still to be assessed. Determining the degree of conservation of this regulatory network between plants from strategy I and strategy II, and between annual and perennial species, is still on its own a question that deserves to be addressed.

How plants sense iron status and switch on or repress the downstream regulatory network to regulate iron uptake, translocation, storage or assimilation has remained elusive. To date, haemerythrin E3-ubiquitin ligases are the main candidates (Rodríguez-Celma et al., 2019a). These E3-ubiquitin ligases are induced in response to iron deficiency, participate to the degradation of key bHLH TFs (i.e. clade IVc and FIT/bHLH29) and are destabilized upon iron binding at their haemerythrin motifs. IDEF1, the rice iron binding TF mentioned earlier, was also proposed as a potential iron sensor (Kobayashi et al., 2012; Kobayashi et al., 2009; Kobayashi et al., 2007). The characterization of IDEF1 functional homologs in non-grass species would be an additional element in support of this later hypothesis. Another important question concerns the regulation of the most upstream bHLH TFs. For instance, the main challenges would be to determine how the expression of clade IVc bHLH is controlled and to identify which protein kinase modulates URI/bHLH121 activity.

Recently, epigenetic regulation has emerged as playing important role in the control of iron homeostasis, by regulating DNA accessibility to promoters, and thus the expression of both TFs and iron uptake genes. This is for instance the case in Arabidopsis with the irondependent deposition of repressive marks on $\mathrm{H} 3$ histone (i.e. $\mathrm{H} 3 \mathrm{~K} 27 \mathrm{~m} 3$ ) at the promoter loci of FIT/bHLH29, IRT1 and FRO2 (Park et al., 2019). This additional layer of regulation in the transcriptional networks controlling iron homeostasis raises questions on the importance and the significance of such regulatory mechanism in this process.

Within this review, it is possible to grasp the extent of the complexity of the regulatory networks that regulate iron homeostasis in plants. Nevertheless, another level of complexity still to be investigated concerns the apparent redundancies existing between several TFs, the localization of their target genes, and thus the physiological functions that are controlled. This will be achieved by the in depth study of the tissue and cellular localization of the 
different TFs as well as the proteins involved in their regulation. Such knowledge is necessary to fully decrypt and understand the dynamics of this regulatory process. Unfortunately, such data are only available for a few of these proteins (Long et al., 2010; Gao et al., 2020a; Samira et al., 2018).

In conclusion, much work still lies ahead to fully comprehend the transcriptional regulatory network that regulate iron homeostasis in plants, which might offer novel opportunities for improving plant growth and health and for generating iron-fortified crops. 


\section{Acknowledgements}

This work was supported by grants from the "Agence Nationale de la Recherche (ANR)" to C.D. Support was provided by the China Scholarship Council to F.G. We thank Florence Vignols for help in preparing this article.

\section{Author Contribution}

FG and CD wrote the manuscript 


\section{References}

\section{Bashir K, Ishimaru Y, Shimo H, Kakei Y, Senoura T, Takahashi R, Sato Y, Sato Y,}

Uozumi N, Nakanishi H, Nishizawa NK. 2011. Rice phenolics efflux transporter 2 (PEZ2) plays an important role in solubilizing apoplasmic iron. Soil Science and Plant Nutrition 57, 803-812.

Briat J-F, Dubos C, Gaymard F. 2015. Iron nutrition, biomass production, and plant product quality. Trends in Plant Science 20, 33-40.

Bruex A, Kainkaryam RM, Wieckowski Y, Kang YH, Bernhardt C, Xia Y, Zheng X, Wang JY, Lee MM, Benfey P. 2012. A gene regulatory network for root epidermis cell differentiation in Arabidopsis. PLoS genetics 8, e1002446.

Brumbarova T, Bauer P, Ivanov R. 2015. Molecular mechanisms governing Arabidopsis iron uptake. Trends in Plant Science 20, 124-133.

Camaschella C. 2015. Iron-deficiency anemia. New England journal of medicine 372, 1832-1843.

Carretero-Paulet L, Galstyan A, Roig-Villanova I, Martínez-García JF, BilbaoCastro JR, Robertson DL. 2010. Genome-wide classification and evolutionary analysis of the bHLH family of transcription factors in Arabidopsis, poplar, rice, moss, and algae. Plant Physiology 153, 1398-1412.

Colangelo EP, Guerinot ML. 2004. The essential basic helix-loop-helix protein FIT1 is required for the iron deficiency response. The Plant Cell 16, 3400-3412.

Coleto I, Bejarano I, Marín-Peña AJ, Medina J, Rioja C, Burow M, Marino D. Arabidopsis thaliana transcription factors MYB28 AND MYB29 shape ammonium stress responses by regulating fe homeostasis. New Phytol doi: 10.1111/nph.16918. 
Colombo C, Iorio ED, Liu Q, Jiang Z, Barrón V. 2018. Iron Oxide Nanoparticles in Soils: Environmental and Agronomic Importance. Journal of Nanoscience and Nanotechnology, 18:761.

Connorton JM, Balk J, Rodríguez-Celma J. 2017. Iron homeostasis in plants-a brief overview. Metallomics 9, 813-823.

Cui Y, Chen C-L, Cui M, Zhou W-J, Wu H-L, Ling H-Q. 2018. Four IVa bHLH transcription factors are novel interactors of FIT and mediate JA inhibition of iron uptake in Arabidopsis. Molecular plant 11, 1166-1183.

Du J, Huang Z, Wang B, Sun H, Chen C, Ling H-Q, Wu H. 2015. SlbHLH068 interacts with FER to regulate the iron-deficiency response in tomato. Annals of botany 116, 23-34.

Fourcroy P, Sisó-Terraza P, Sudre D, Savirón M, Reyt G, Gaymard F, Abadía A, Abadia J, Álvarez-Fernández A, Briat JF. 2014. Involvement of the ABCG 37 transporter in secretion of scopoletin and derivatives by Arabidopsis roots in response to iron deficiency. New Phytologist 201, 155-167.

Fourcroy P, Tissot N, Gaymard F, Briat J-F, Dubos C. 2016. Facilitated Fe nutrition by phenolic compounds excreted by the Arabidopsis ABCG37/PDR9 transporter requires the IRT1/FRO2 high-affinity root $\mathrm{Fe} 2+$ transport system. Molecular plant $\mathbf{9}$, 485-488.

Gao F, Robe K, Bettembourg M, Navarro N, Rofidal V, Santoni V, Gaymard F, Vignols F, Roschzttardtz H, Izquierdo E. 2020a. The transcription factor bHLH121 interacts with bHLH105 (ILR3) and its closest homologs to regulate iron homeostasis in Arabidopsis. The Plant Cell 32, 508-524.

Gao F, Robe K, Dubos C. 2020b. Further insights into the role of bHLH121 in the regulation of iron homeostasis in Arabidopsis thaliana. Plant Signaling and Behavior, 1795582.

Gao F, Robe K, Gaymard F, Izquierdo E, Dubos C. 2019. The transcriptional control of iron homeostasis in plants: a tale of bHLH transcription factors? Frontiers in plant science 10, 6 . 
Gollhofer J, Timofeev R, Lan P, Schmidt W, Buckhout TJ. 2014. Vacuolar-irontransporter1-like proteins mediate iron homeostasis in Arabidopsis. PLoS One 9, e110468.

Gratz R, Manishankar P, Ivanov R, Köster P, Mohr I, Trofimov K, Steinhorst L, Meiser J, Mai H-J, Drerup M. 2019. CIPK11-dependent phosphorylation modulates FIT activity to promote Arabidopsis iron acquisition in response to calcium signaling. Developmental cell 48, 726-740. e710.

Grillet L, Lan P, Li W, Mokkapati G, Schmidt W. 2018. IRON MAN is a ubiquitous family of peptides that control iron transport in plants. Nature plants 4, 953-963.

Guerinot ML, Yi Y. 1994. Iron: nutritious, noxious, and not readily available. Plant Physiology 104, 815-820.

Hänsch R, Mendel RR. 2009. Physiological functions of mineral micronutrients (cu, Zn, $\mathrm{Mn}, \mathrm{Fe}, \mathrm{Ni}, \mathrm{Mo}, \mathrm{B}, \mathrm{cl})$. Current Opinion in Plant Biology 12, 259-266.

Heim MA, Jakoby M, Werber M, Martin C, Weisshaar B, Bailey PC. 2003. The basic helix-loop-helix transcription factor family in plants: a genome-wide study of protein structure and functional diversity. Molecular biology and evolution 20, 735747.

Hindt MN, Akmakjian GZ, Pivarski KL, Punshon T, Baxter I, Salt DE, Guerinot ML. 2017. BRUTUS and its paralogs, BTS LIKE1 and BTS LIKE2, encode important negative regulators of the iron deficiency response in Arabidopsis thaliana. Metallomics 9, 876-890.

Hirayama T, Lei GJ, Yamaji N, Nakagawa N, Ma JF. 2018. The putative peptide gene FEP1 regulates iron deficiency response in Arabidopsis. Plant and Cell Physiology 59, $1739-1752$.

Huang D, Dai W. 2015. Molecular characterization of the basic helix-loop-helix (bHLH) genes that are differentially expressed and induced by iron deficiency in Populus. Plant cell reports 34, 1211-1224.

Inoue H, Kobayashi T, Nozoye T, Takahashi M, Kakei Y, Suzuki K, Nakazono M, Nakanishi H, Mori S, Nishizawa NK. 2009. Rice OsYSL15 is an iron-regulated iron (III)-deoxymugineic acid transporter expressed in the roots and is essential for iron 
uptake in early growth of the seedlings. Journal of Biological Chemistry 284, 34703479.

Ishimaru Y, Suzuki M, Tsukamoto T, Suzuki K, Nakazono M, Kobayashi T, Wada Y, Watanabe S, Matsuhashi S, Takahashi M. 2006. Rice plants take up iron as an Fe3+-phytosiderophore and as Fe2+. The Plant Journal 45, 335-346.

Jakoby M, Wang H-Y, Reidt W, Weisshaar B, Bauer P. 2004. FRU (BHLH029) is required for induction of iron mobilization genes in Arabidopsis thaliana. FEBS letters 577, 528-534.

Kagale S, Rozwadowski K. 2011. EAR motif-mediated transcriptional repression in plants: an underlying mechanism for epigenetic regulation of gene expression. Epigenetics 6, 141-146.

Kim SA, LaCroix IS, Gerber SA, Guerinot ML. 2019. The iron deficiency response in Arabidopsis thaliana requires the phosphorylated transcription factor URI. Proceedings of the National Academy of Sciences 116, 24933-24942.

Kobayashi T. 2019.Understanding the Complexity of Iron Sensing and Signaling Cascades in Plants. Plant and Cell Physiology 60, 1440-1446.

Kobayashi T, Itai RN, Aung MS, Senoura T, Nakanishi H, Nishizawa NK. 2012. The rice transcription factor IDEF1 directly binds to iron and other divalent metals for sensing cellular iron status. The Plant Journal 69, 81-91.

Kobayashi T, Itai RN, Ogo Y, Kakei Y, Nakanishi H, Takahashi M, Nishizawa NK. 2009. The rice transcription factor IDEF1 is essential for the early response to iron deficiency, and induces vegetative expression of late embryogenesis abundant genes. The Plant Journal 60, 948-961.

Kobayashi T, Nagasaka S, Senoura T, Itai RN, Nakanishi H, Nishizawa NK. 2013. Iron-binding haemerythrin RING ubiquitin ligases regulate plant iron responses and accumulation. Nature communications 4, 1-12.

Kobayashi T, Nakanishi Itai R, Nishizawa NK. 2014. Iron deficiency responses in rice roots. Rice (N Y) 7, 27.

Kobayashi T, Nishizawa NK. 2012. Iron uptake, translocation, and regulation in higher plants. Annual Review of Plant Biology 63, 131-152. 
Kobayashi T, Nozoye T, Nishizawa NK. 2019. Iron transport and its regulation in plants. Free Radical Biology and Medicine 133, 11-20.

Kobayashi T, Ogo Y, Itai RN, Nakanishi H, Takahashi M, Mori S, Nishizawa NK. 2007. The transcription factor IDEF1 regulates the response to and tolerance of iron deficiency in plants. Proceedings of the National Academy of Sciences 104, 1915019155.

Kobayashi T, Ozu A, Kobayashi S, An G, Jeon J-S, Nishizawa NK. 2019. OsbHLH058 and OsbHLH059 transcription factors positively regulate iron deficiency responses in rice. Plant Molecular Biology 101, 471-486.

Kroh GE, Pilon M. 2019. Connecting the negatives and positives of plant iron homeostasis. New Phytologist 223,1052-1055.

Le CTT, Brumbarova T, Ivanov R, Stoof C, Weber E, Mohrbacher J, Fink-Straube C, Bauer P. 2016. Zinc finger of Arabidopsis thaliana12 (ZAT12) interacts with FERlike iron deficiency-induced transcription factor (FIT) linking iron deficiency and oxidative stress responses. Plant Physiology 170, 540-557.

Lei R, Li Y, Cai Y, Li C, Pu M, Lu C, Yang Y, Liang G. 2020. bHLH121 Functions as a Direct Link that Facilitates the Activation of FIT by bHLH IVc Transcription Factors for Maintaining Fe Homeostasis in Arabidopsis. Molecular plant 13, 634-649.

Li L, Gao W, Peng Q, Zhou B, Kong Q, Ying Y, Shou H. 2018. Two soybean bHLH factors regulate response to iron deficiency. Journal of integrative plant biology $\mathbf{6 0}$, 608-622.

Li Q, Chen L, Yang A. 2019. The Molecular Mechanisms Underlying Iron Deficiency Responses in Rice. International Journal of Molecular Sciences 21, 43.

Li X, Zhang H, Ai Q, Liang G, Yu D. 2016. Two bHLH transcription factors, bHLH34 and bHLH104, regulate iron homeostasis in Arabidopsis thaliana. Plant Physiology 170, 2478-2493.

Li Y-Y, Sui X-Y, Yang J-S, Xiang X-H, Li Z-Q, Wang Y-Y, Zhou Z-C, Hu R-S, Liu D. 2020. A novel bHLH transcription factor, NtbHLH1, modulates iron homeostasis in tobacco (Nicotiana tabacum L.). Biochemical and Biophysical Research Communications 522, 233-239. 
Liang G, Zhang H, Li X, Ai Q, Yu D. 2017. bHLH transcription factor bHLH115 regulates iron homeostasis in Arabidopsis thaliana. Journal of experimental botany 68 , 1743-1755.

Liang G, Zhang H, Li Y, Pu M, Yang Y, Li C, Lu C, Xu P, Yu D. 2020. Oryza sativa Fer-like Fe deficiency-induced transcription factor (OsFIT/OsbHLH156) interacts with OsIRO2 to regulate iron homeostasis. Journal of integrative plant biology 62, 668-689.

Ling H-Q, Bauer P, Bereczky Z, Keller B, Ganal M. 2002. The tomato fer gene encoding a bHLH protein controls iron-uptake responses in roots. Proceedings of the National Academy of Sciences 99, 13938-13943.

Lingam S, Mohrbacher J, Brumbarova T, Potuschak T, Fink-Straube C, Blondet E, Genschik P, Bauer P. 2011. Interaction between the bHLH transcription factor FIT and ETHYLENE INSENSITIVE3/ETHYLENE INSENSITIVE3-LIKE1 reveals molecular linkage between the regulation of iron acquisition and ethylene signaling in Arabidopsis. The Plant Cell 23, 1815-1829.

Liu W, Karemera NU, Wu T, Yang Y, Zhang X, Xu X, Wang Y, Han Z. 2017a. The ethylene response factor AtERF4 negatively regulates the iron deficiency response in Arabidopsis thaliana. PLoS One 12, e0186580.

Liu W, Li Q, Wang Y, Wu T, Yang Y, Zhang X, Han Z, Xu X. 2017b. Ethylene response factor AtERF72 negatively regulates Arabidopsis thaliana response to iron deficiency. Biochemical and Biophysical Research Communications 491, 862-868.

Liu W, Wu T, Li Q, Zhang X, Xu X, Li T, Han Z, Wang Y. 2018. An ethylene response factor (MxERF4) functions as a repressor of $\mathrm{Fe}$ acquisition in Malus xiaojinensis. Scientific reports 8, 1-13.

Lockhart J. 2020. Personal Trainer: bHLH121 Functions Upstream of a Transcriptional Network of Heavy Lifters Involved in Balancing Iron Levels. The Plant Cell 32, 293294.

Long TA, Tsukagoshi H, Busch W, Lahner B, Salt DE, Benfey PN. 2010. The bHLH transcription factor POPEYE regulates response to iron deficiency in Arabidopsis roots. The Plant Cell 22, 2219-2236. 
Marschner H, Römheld V. 1994. Strategies of plants for acquisition of iron. Plant and soil 165, 261-274.

Martín Barranco A, Spielmann J, Dubeaux G, Vert G, Zelazny E. 2020. Dynamic control of the high-affinity iron uptake complex in root epidermal cells. Plant Physiology, doi:10.1104/pp.20.00234

Maurer F, Arcos MAN, Bauer P. 2014. Responses of a triple mutant defective in three iron deficiency-induced basic helix-loop-helix genes of the subgroup $\mathrm{Ib}$ to iron deficiency and salicylic acid. PLoS One 9, e99234.

Murata Y, Ma JF, Yamaji N, Ueno D, Nomoto K, Iwashita T. 2006. A specific transporter for iron (III)-phytosiderophore in barley roots. The Plant Journal 46, 563572 .

Ogo Y, Itai RN, Nakanishi H, Inoue H, Kobayashi T, Suzuki M, Takahashi M, Mori S, Nishizawa NK. 2006. Isolation and characterization of IRO2, a novel ironregulated bHLH transcription factor in graminaceous plants. Journal of experimental botany $\mathbf{5 7}, 2867-2878$.

Ogo Y, Kobayashi T, Itai RN, Nakanishi H, Kakei Y, Takahashi M, Toki S, Mori S, Nishizawa NK. 2008. A novel NAC transcription factor, IDEF2, that recognizes the iron deficiency-responsive element 2 regulates the genes involved in iron homeostasis in plants. Journal of Biological Chemistry 283, 13407-13417.

Ogo Y, Nakanishi Itai R, Nakanishi H, Kobayashi T, Takahashi M, Mori S, Nishizawa NK. 2007. The rice bHLH protein OsIRO2 is an essential regulator of the genes involved in Fe uptake under Fe-deficient conditions. The Plant Journal 51, 366377 .

Palmer CM, Hindt MN, Schmidt H, Clemens S, Guerinot ML. 2013. MYB10 and MYB72 are required for growth under iron-limiting conditions. PLoS genetics 9, e1003953.

Park EY, Tsuyuki KM, Hu F, Lee J, Jeong J. 2019. PRC2-mediated H3K27me3 contributes to transcriptional regulation of FIT-dependent iron deficiency response. Frontiers in plant science 10, 627. 
Qi Y, Wang S, Shen C, Zhang S, Chen Y, Xu Y, Liu Y, Wu Y, Jiang D. 2012. OsARF12, a transcription activator on auxin response gene, regulates root elongation and affects iron accumulation in rice (Oryza sativa). New Phytologist 193, 109-20.

Ramamurthy RK, Waters BM. 2017. Mapping and characterization of the fefe gene that controls iron uptake in melon (Cucumis melo L.). Frontiers in plant science 8, 1003.

Robe K, Conejero G, Gao F, Fourcroy P, Lefebvre-Legendre L, Sylvestre-Gonon E, Rofidal V, Hem S, Rouhier N, Barberon M, Hecker A, Gaymard F, Izquierdo E, Dubos C. 2020. Coumarin accumulation and trafficking in Arabidopsis thaliana: a complex and dynamic process. New Phytologist In press.

Rodríguez-Celma J, Chou H, Kobayashi T, Long TA, Balk J. 2019a. Hemerythrin E3 ubiquitin ligases as negative regulators of iron homeostasis in plants. Frontiers in plant science 10, 98 .

Rodríguez-Celma J, Connorton JM, Kruse I, Green RT, Franceschetti M, Chen YT, Cui Y, Ling H-Q, Yeh K-C, Balk J. 2019b. Arabidopsis BRUTUS-LIKE E3 ligases negatively regulate iron uptake by targeting transcription factor FIT for recycling. Proceedings of the National Academy of Sciences 116, 17584-17591.

Roschzttardtz H, Gaymard F, Dubos C. 2020. Transcriptional Regulation of Iron Distribution in Seeds: A Perspective. Frontiers in Plant Science 11, 725.

Samira R, Li B, Kliebenstein D, Li C, Davis E, Gillikin JW, Long TA. 2018. The bHLH transcription factor ILR3 modulates multiple stress responses in Arabidopsis. Plant Molecular Biology 97, 297-309.

Santi S, Schmidt W. 2009. Dissecting iron deficiency-induced proton extrusion in Arabidopsis roots. New Phytologist 183, 1072-1084.

Schwarz B, Bauer P. 2020. FIT, a regulatory hub for iron deficiency and stress signaling in roots, and FIT-dependent and -independent gene signatures. Journal of Experimental Botany 71, 1694-1705.

Selote D, Samira R, Matthiadis A, Gillikin JW, Long TA. 2015. Iron-binding E3 ligase mediates iron response in plants by targeting basic helix-loop-helix transcription factors. Plant Physiology 167, 273-286. 
Sharma R, Yeh KC. 2020. The dual benefit of a dominant mutation in Arabidopsis IRON DEFICIENCY TOLERANT1 for iron biofortification and heavy metal phytoremediation. Plant biotechnology journal 18, 1200-1210.

Shen J, Xu X, Li T, Cao D, Han Z. 2008. An MYB transcription factor from Malus xiaojinensis has a potential role in iron nutrition. Journal of integrative plant biology 50, 1300-1306.

Spielmann J, Vert G. 2020. The many facets of protein ubiquitination and degradation in plant root iron deficiency responses. Journal of Experimental Botany, doi: 10.1093/jxb.

Shen C, Yue R, Sun T, Zhang L, Yang Y, Wang H. 2015. OsARF16, a transcription factor regulating auxin redistribution, is required for iron deficiency response in rice (Oryza sativa L.). Plant Science 231, 148-58.

Sun Y, Li JQ, Yan JY, Yuan JJ, Li GX, Wu YR, Xu JM, Huang RF, Harberd NP, Ding ZJ, Zheng SJ. 2020. Ethylene promotes seed iron storage during Arabidopsis seed maturation via ERF95 transcription factor. Journal of Integrative Plant Biology 62, 1193-1212.

Stringlis IA, Yu K, Feussner K, de Jonge R, Van Bentum S, Van Verk MC, Berendsen RL, Bakker PA, Feussner I, Pieterse CM. 2018. MYB72-dependent coumarin exudation shapes root microbiome assembly to promote plant health. Proceedings of the National Academy of Sciences 115, E5213-E5222.

Tanabe N, Noshi M, Mori D, Nozawa K, Tamoi M, Shigeoka S. 2019. The basic helixloop-helix transcription factor, bHLH11 functions in the iron-uptake system in Arabidopsis thaliana. Journal of plant research 132, 93-105.

Tissot N, Robe K, Gao F, Grant-Grant S, Boucherez J, Bellegarde F, Maghiaoui A, Marcelin R, Izquierdo E, Benhamed M. 2019. Transcriptional integration of the responses to iron availability in Arabidopsis by the bHLH factor ILR3. New Phytologist 223, 1433-1446.

Touraine B, Vignols F, Przybyla-Toscano J, Ischebeck T, Dhalleine T, Wu H-C, Magno C, Berger N, Couturier J, Dubos C. 2019. Iron-sulfur protein NFU2 is required for branched-chain amino acid synthesis in Arabidopsis roots. Journal of experimental botany 70, 1875-1889. 
Trofimov K, Ivanov R, Eutebach M, Acaroglu B, Mohr I, Bauer P, Brumbarova T. 2019. Mobility and localization of the iron deficiency-induced transcription factor bHLH039 change in the presence of FIT. Plant Direct 3, e00190.

Wang F-P, Wang X-F, Zhang J, Ma F, Hao Y-J. 2018. MdMYB58 modulates Fe homeostasis by directly binding to the MdMATE43 promoter in plants. Plant and Cell Physiology 59, 2476-2489.

Wang L, Ying Y, Narsai R, Ye L, Zheng L, Tian J, Whelan J, Shou H. 2013a. Identification of OsbHLH133 as a regulator of iron distribution between roots and shoots in Oryza sativa. Plant, cell \& environment 36, 224-236.

Wang N, Cui Y, Liu Y, Fan H, Du J, Huang Z, Yuan Y, Wu H, Ling H-Q. 2013b. Requirement and functional redundancy of $\mathrm{Ib}$ subgroup bHLH proteins for iron deficiency responses and uptake in Arabidopsis thaliana. Molecular plant 6, 503-513.

Wang S, Li L, Ying Y, Wang J, Shao JF, Yamaji N, Whelan J, Ma JF, Shou H. 2020. A transcription factor OsbHLH156 regulates Strategy II iron acquisition through localising IRO2 to the nucleus in rice. New Phytologist 225, 1247-1260.

Wu H, Ling H-Q. 2019. FIT-Binding Proteins and Their Functions in the Regulation of Fe Homeostasis. Frontiers in plant science 10, 844.

Xu Z, Liu X, He X, Xu L, Huang Y, Shao H, Zhang D, Tang B, Ma H. 2017. The soybean basic helix-loop-helix transcription factor ORG3-Like enhances cadmium tolerance via increased iron and reduced cadmium uptake and transport from roots to shoots. Frontiers in plant science 8, 1098.

Yan JY, Li CX, Sun L, Ren JY, Li GX, Ding ZJ, Zheng SJ. 2016. A WRKY transcription factor regulates Fe translocation under Fe deficiency. Plant Physiology 171, 2017-2027.

Yin L, Wang Y, Yan M, Zhang X, Pan H, Xu X, Han Z. 2013. Molecular cloning, polyclonal antibody preparation, and characterization of a functional iron-related transcription factor IRO2 from Malus xiaojinensis. Plant physiology and biochemistry 67, 63-70.

Yin L, Wang Y, Yuan M, Zhang X, Xu X, Han Z. 2014. Characterization of MxFIT, an iron deficiency induced transcriptional factor in Malus xiaojinensis. Plant physiology and biochemistry 75, 89-95. 
Yuan Y, Wu H, Wang N, Li J, Zhao W, Du J, Wang D, Ling H-Q. 2008. FIT interacts with AtbHLH38 and AtbHLH39 in regulating iron uptake gene expression for iron homeostasis in Arabidopsis. Cell research 18, 385-397.

Yuan YX, Zhang J, Wang DW, Ling HQ. 2005. AtbHLH29 of Arabidopsis thaliana is a functional ortholog of tomato FER involved in controlling iron acquisition in strategy I plants. Cell research 15, 613-621.

Zamioudis C, Hanson J, Pieterse CMJ. 2014. $\beta$-Glucosidase BGLU42 is a MYB72dependent key regulator of rhizobacteria-induced systemic resistance and modulates iron deficiency responses in Arabidopsis roots. New Phytologist 204, 368-379.

Zhang G, Liu W, Feng Y, Li D, Li K, Sun Q, Zhai L, Wu T, Zhang X, Xu X. 2020a. Ethylene Response Factors MbERF4 and MbERF72 Suppress Iron Uptake in Woody Apple Plants by Modulating Rhizosphere pH. Plant and Cell Physiology 61, 699-711.

Zhang H, Li Y, Pu M, Xu P, Liang G, Yu D. 2020b. Oryza sativa POSITIVE REGULATOR OF IRON DEFICIENCY RESPONSE 2 (OsPRI2) and OsPRI3 are involved in the maintenance of Fe homeostasis. Plant, cell \& environment 43, 261274.

Zhang H, Li Y, Yao X, Liang G, Yu D. 2017. Positive regulator of iron homeostasis1, OsPRI1, facilitates iron homeostasis. Plant Physiology 175, 543-554.

Zhang J, Liu B, Li M, Feng D, Jin H, Wang P, Liu J, Xiong F, Wang J, Wang H-B. 2015. The bHLH transcription factor bHLH104 interacts with IAA-LEUCINE RESISTANT3 and modulates iron homeostasis in Arabidopsis. The Plant Cell 27, 787-805.

Zhang L, Nakanishi Itai R, Yamakawa T, Nakanishi H, Nishizawa NK, Kobayashi T. 2014. The Bowman-Birk Trypsin Inhibitor IBP1 Interacts with and Prevents Degradation of IDEF1 in Rice. Plant Molecular Biology Reporter 32, 841-851.

Zhang X, Wang C, Zhang Y, Sun Y, Mou Z. 2012. The Arabidopsis mediator complex subunit16 positively regulates salicylate-mediated systemic acquired resistance and jasmonate/ethylene-induced defense pathways. The Plant Cell 24, 4294-4309.

Zhao M, Song A, Li P, Chen S, Jiang J, Chen F. 2014. A bHLH transcription factor regulates iron intake under Fe deficiency in chrysanthemum. Scientific reports $\mathbf{4}$, 6694. 
Zhao Q, Ren Y-R, Wang Q-J, Wang X-F, You C-X, Hao Y-J. 2016a. Ubiquitinationrelated MdBT scaffold proteins target a bHLH transcription factor for iron homeostasis. Plant Physiology 172, 1973-1988.

Zhao Q, Ren YR, Wang QJ, Yao YX, You CX, Hao YJ. 2016b. Overexpression of Mdb HLH 104 gene enhances the tolerance to iron deficiency in apple. Plant biotechnology journal 14, 1633-1645.

Zheng L, Ying Y, Wang L, Wang F, Whelan J, Shou H. 2010. Identification of a novel iron regulated basic helix-loop-helix protein involved in $\mathrm{Fe}$ homeostasis in Oryza sativa. BMC plant biology 10, 166.

Zhu XF, Wu Q, Meng YT, Tao Y, Shen RF. 2020. AtHAP5A regulates iron translocation in iron-deficient Arabidopsis thaliana. Journal of Integrative Plant Biology doi.org/10.1111/jipb.12984

Zhou L-J, Zhang C-L, Zhang R-F, Wang G-L, Li Y-Y, Hao Y-J. 2019. The SUMO E3 ligase MdSIZ1 targets MdbHLH104 to regulate plasma membrane H+-ATPase activity and iron homeostasis. Plant Physiology 179, 88-106. 
Table 1. bHLH TFs involved in the regulation of iron homeostasis in plants.

\begin{tabular}{|c|c|c|c|c|}
\hline Name & Clade & Species & Interacting proteins & Reference \\
\hline AtbHLH38* & $\mathrm{Ib}$ & A. thaliana & FIT/AtbHLH29, DELLAs & Yuan et al., 2008; Wang et al., 2013 \\
\hline AtbHLH39* & $\mathrm{Ib}$ & A. thaliana & FIT/AtbHLH29, DELLAs & Yuan et al., 2008; Wang et al., 2013 \\
\hline AtbHLH100* & $\mathrm{Ib}$ & A. thaliana & FIT/AtbHLH29 & Wang et al., 2013 \\
\hline AtbHLH101* & $\mathrm{Ib}$ & A. thaliana & FIT/AtbHLH29 & Wang et al., 2013 \\
\hline FIT/AtbHLH29 * & IIIa & A. thaliana & $\begin{array}{l}\text { AtbHLH38, AtbHLH39, AtbHLH100, } \\
\text { AtbHLH101, AtbHLH18, AtbHLH19, } \\
\text { AtbHLH20, AtbHLH25, BTSL1, } \\
\text { BTSL2, CIPK11, DELLAs, EIL1, } \\
\text { EIN3, MED16, ZAT12 }\end{array}$ & $\begin{array}{l}\text { Colangelo and Guerinot, 2004; } \\
\text { Jakoby et al., 2004; Yuan et al., } 2005\end{array}$ \\
\hline MYC2/AtbHLH6 & IIIe & A. thaliana & & Cui et al., 2018 \\
\hline AtbHLH18* & IVa & A. thaliana & FIT/AtbHLH29 & Cui et al., 2018 \\
\hline AtbHLH19* & IVa & A. thaliana & FIT/AtbHLH29 & Cui et al., 2018 \\
\hline AtbHLH20* & IVa & A. thaliana & FIT/AtbHLH29 & Cui et al., 2018 \\
\hline AtbHLH25* & IVa & A. thaliana & FIT/AtbHLH29 & Cui et al., 2018 \\
\hline AtbHLH11 & $\mathrm{IVb}$ & A. thaliana & $\begin{array}{l}\text { IDT1/AtbHLH34, AtbHLH104, } \\
\text { ILR3/AtbHLH105, AtbHLH115 }\end{array}$ & Tanabe et al., 2019 \\
\hline PYE/AtbHLH47 & $\mathrm{IVb}$ & A. thaliana & ILR3/AtbHLH105, AtbHLH115 & Long et al., 2010 \\
\hline URI/AtbHLH121 & $\mathrm{IVb}$ & A. thaliana & $\begin{array}{l}\text { IDT1/AtbHLH34, AtbHLH104, } \\
\text { ILR3/AtbHLH105, AtbHLH115 }\end{array}$ & $\begin{array}{l}\text { Gao et al., 2020b; Kim et al., 2019; } \\
\text { Lei et al., } 2020\end{array}$ \\
\hline IDT1/AtbHLH34 & IVc & A. thaliana & IDT1/AtbHLH34, AtbHLH104, & Li et al., 2016 \\
\hline
\end{tabular}




\begin{tabular}{|c|c|c|c|c|}
\hline & & & $\begin{array}{l}\text { ILR3/AtbHLH105, AtbHLH115, } \\
\text { AtbHLH11, URI/bHLH121 }\end{array}$ & \\
\hline AtbHLH104 & IVc & A. thaliana & $\begin{array}{l}\text { IDT1/AtbHLH34, AtbHLH104, } \\
\text { ILR3/AtbHLH105, AtbHLH115, } \\
\text { AtbHLH11, URI/ bHLH121 }\end{array}$ & Zhang et al., 2015 \\
\hline ILR3/AtbHLH105 & IVc & A. thaliana & $\begin{array}{l}\text { IDT1/AtbHLH34, AtbHLH104, } \\
\text { ILR3/AtbHLH105, AtbHLH115, } \\
\text { AtbHLH11, PYE/ bHLH47, } \\
\text { URI/bHLH121, BTS }\end{array}$ & Zhang et al., 2015 \\
\hline AtbHLH115 & IVc & A. thaliana & $\begin{array}{l}\text { IDT1/AtbHLH34, AtbHLH104, } \\
\text { ILR3/AtbHLH105, AtbHLH115, } \\
\text { AtbHLH11, PYE/ bHLH47, } \\
\text { URI/bHLH121, BTS }\end{array}$ & Liang et al., 2015 \\
\hline OsIRO2/OsbHLH56 & $\mathrm{Ib}$ & O. sativa & OsFIT/OsbHLH156 & Ogo et al., 2006 \\
\hline OsIRO3/OsbHLH63 & $\mathrm{IVb}$ & O. sativa & & Zheng et al., 2010 \\
\hline OsFIT/OsbHLH156 & IIIa & O. sativa & OsIRO2/OsbHLH56 & Liang et al., 2020; Wang et al., 2020 \\
\hline OsPRI1/OsbHLH60 & IVc & O. sativa & OsHRZ1 & Zhang et al., 2017 \\
\hline OsPRI2/OsbHLH58 & IVc & O. sativa & OsHRZ1 & Kobayashi et al., 2019 \\
\hline OsPRI3/OsbHLH59 & IVc & O. sativa & OsHRZ1 & Kobayashi et al., 2019 \\
\hline OsbHLH133 & VIIIc & O. sativa & & Wang et al., 2013 \\
\hline GmbHLH57 & IIIa & G. $\max$ & GmbHLH300 & Li et al., 2018 \\
\hline GmbHLH300 & $\mathrm{Ib}$ & G. $\max$ & GmbHLH57 & Li et al., 2018 \\
\hline FER & IIIa & S. lycopersicum & SlbHLH68 & Ling et al., 2002 \\
\hline SlbHLH68 & $\mathrm{Ib}$ & S. lycopersicum & FER & Du et al., 2015 \\
\hline MxIRO2 & $\mathrm{Ib}$ & M. xiaojinensis & & Yin et al., 2013 \\
\hline MxFIT & IIIa & M. xiaojinensis & MxERF4 & Yin et al., 2014 \\
\hline PtFIT & IIIIa & P. tremula & & Huang and Dai, 2015 \\
\hline PtIRO & $\mathrm{Ib}$ & P. tremula & & Huang and Dai, 2015 \\
\hline SAT1/MdbHLH18 & IVa & M. domestica & MdMYB58 & Wang et al., 2018 \\
\hline
\end{tabular}




\begin{tabular}{|l|l|l|l|l|}
\hline MdbHLH104 & IVc & M. domestica & $\begin{array}{l}\text { MdBT1, MdBT2, MdbHLH104, } \\
\text { MdbHLH105, MdbHLH115, } \\
\text { MdbHLH11, MdbHLH121, MdPYE }\end{array}$ & Zhao et al., 2016b \\
\hline NtbHLH1 & IVb & N. tabacum & & Li et al., 2020 \\
\hline CmbHLH1 & IVc & C. morifolium & & Zhao et al., 2014 \\
\hline FEFE/CmbHLH38 & Ib & C. melo & CmFIT & Ramamurthy and Waters, 2017 \\
\hline GmORG3 & Ib & G. max & & Xu et al., 2017 \\
\hline
\end{tabular}

*: for details on posttranslational regulation of FIT activity, see Wu and Ling, 2020 and Schwarz and Bauer, 2020. 
Table 2. TFs other than bHLHs involved in the regulation of iron homeostasis in plants.

\begin{tabular}{|c|c|c|c|c|}
\hline TF family & Gene name & Species & Interacting proteins & Reference \\
\hline $\mathrm{ABI} / \mathrm{VP} 1$ & IDEF1 & O. sativa & IBP1 & Kobayashi et al., 2007 \\
\hline ARF & OsARF12 & O. sativa & & Qi et al., 2012 \\
\hline ARF & OsARF16 & O. sativa & & Shen et al., 2016 \\
\hline $\mathrm{C} 2 \mathrm{H} 2$ & ZAT12* & A. thaliana & FIT/bHLH29 & Le et al., 2016 \\
\hline EIL & EIN3* & A. thaliana & FIT/bHLH29 & Lingam et al., 2011 \\
\hline EIL & EIL1* & A. thaliana & FIT/bHLH29 & Lingam et al., 2011 \\
\hline ERF & ERF4 & A. thaliana & & Liu et al., 2017a \\
\hline ERF & ERF72 & A. thaliana & & Liu et al., 2017b \\
\hline ERF & ERF95 & A. thaliana & & Sun et al., 2020 \\
\hline ERF & MxERF4 & M. xiaojinensis & MxFIT & Liu et al., 2018 \\
\hline ERF & MbERF4 & M. baccata & MbERF72 & Zhang et al., 2020a \\
\hline ERF & MbERF72 & M. baccata & MbERF4 & Zhang et al., 2020a \\
\hline MYB (R2R3) & MYB10 & A. thaliana & & Palmer et al., 2013 \\
\hline MYB (R2R3) & MYB28 & A. thaliana & & Coleto et al., 2020 \\
\hline MYB (R2R3) & MYB29 & A. thaliana & & Coleto et al., 2020 \\
\hline MYB (R2R3) & \begin{tabular}{|l|} 
MYB72 \\
\end{tabular} & A. thaliana & & Palmer et al., 2013 \\
\hline MYB (R2R3) & \begin{tabular}{|l|} 
MdMYB58 \\
\end{tabular} & M. domestica & MdSAT1/MdbHLH18 & Wang et al., 2018 \\
\hline MYB (R2R3) & MxMYB1 & M. xiaojinensis & & Shen et al., 2008 \\
\hline NAC & IDEF2 & O. sativa & & Ogo et al., 2008 \\
\hline NF-YC & HAP5A/NF-YC1 & A. thaliana & & Zhu et al., 2020 \\
\hline WRKY & WRKY46 & A. thaliana & & Yan et al., 2016 \\
\hline
\end{tabular}

*: for details on posttranslational regulation of FIT activity, see Wu and Ling. 2020 and Schwarz and Bauer, 2020. 


\section{Figure legends}

Figure 1. Schematic diagram of iron uptake strategies in Arabidopsis and rice. In Arabidopsis, AHA2 secretes protons into the rhizosphere to increase $\mathrm{Fe}^{3+}$ solubility; PDR9 secretes Fe-mobilizing coumarins (i.e. fraxetin and sideretin) to mobilize and chelate $\mathrm{Fe}^{3+} \cdot \mathrm{Fe}^{3+}$ is reduced into $\mathrm{Fe}^{2+}$ and is subsequently transported into the root epidermal cells by FRO2 and IRT1, respectively. AHA2, FRO2 and IRT1 form a protein complex optimizing Fe acquisition by creating a local environment with low $\mathrm{pH}$ and high $\mathrm{Fe}^{2+}$ concentration. Rice biosynthesizes and secretes DMA (2-deoxy-mugineic acid) to chelate $\mathrm{Fe}^{3+} . \mathrm{Fe}^{3+}$-DMA complexes are transported into root cells via YSL15 and YSL16. In addition, rice also uptakes $\mathrm{Fe}^{2+}$ from the soil through the activity of two $\mathrm{Fe}^{2+}$ transporters, OsIRT1 and OsIRT2 under waterlogged soil condition. The secretion of CA and PCA via the PEZ2 phenolics efflux transporter participates to $\mathrm{Fe}^{3+}$ mobilization and reduction into $\mathrm{Fe}^{2+}$. AHA2: $\mathrm{H}^{+}$-ATPase 2; PDR9: PLEIOTROPIC DRUG RESISTANCE 9/ATP-BINDING CASSETTE G37; FRO2: FERRIC REDUCTION OXIDASE 2; IRT1: IRON-REGULATED TRANSPORTER 1, F6'H1: FERULOYL CoA 6' HYDROXYLASE 1; S8H: SCOPOLETIN 8-HYDROXYLASE; CYP82C4: FRAXETIN 5-HYDROXYLASE; TOM1: TRANSPORTER OF MUGINEIC ACID 1; YSL15/16: YELLOW STRIPE-LIKE 15/16; OsIRT1/2: Rice IRON-REGULATED TRANSPORTER 1/2; PEZ2: PHENOLICS EFFLUX ZERO 2; OsNAS: NICOTIANAMINE SYNTHASE; OSNAAT: NICOTIANAMINE AMINOTRANSFERASE; OsDMAS: DEOXYMUGINEIC ACID SYNTHASE; SAM: S-adenosyl methionine; NA: nicotianamine; CA: caffeic acid; PCA: protocatechuic acid.

Figure 2. Phylogenetic tree of bHLH TFs involved in the regulation of iron homeostasis in plants. The tree was constructed by using the neighbor-joining method with the MEGA software (v10.0.5) using full-length bHLH amino acid sequences. The bootstrap analysis was carried out with 1000 replicates. Sequences were aligned prior to the construction of the phylogenetic tree using the ClustalX software (v2.0.11). The different bHLH clades are designated as previously reported (Heim et al., 2003). Species abbreviations: At, Arabidopsis thaliana; Md, Malus domestica; Nt, Nicotiana tabacum; 
Os, Oryza sativa; $\mathrm{Sl}$, Solanum lycopersicum; Gm, Glycine max; $\mathrm{Mx}$, Malus xiaojinensis; $\mathrm{Cm}$, (CmbHLH1), Chrysanthemum morifolium; Cm (CmbHLH38), Cucumis melo; Pt, Populus tremula.

Figure 3. bHLH TFs network that regulates iron homeostasis in Arabidopsis. Question marks indicate protein complexes for which transcriptional activities have not yet been clearly demonstrated. The colour code for the bHLH TFs refers to the clades described Figure 2. Non bHLH proteins are in grey. EIN3 and EIL1 favor FIT stability whereas MED16 and MED25 mediate the FIT/bHLH29-dependent iron deficiency responses (for additional details on posttranslational regulation of FIT, see Kobayashi, 2019; Schwarz and Bauer, 2020; Spielmann and Vert, 2020; Wu and Ling, 2019). EIN3: ETHYLENE INSENSITIVE 3; EIL1: ETHYLENE INSENSITIVE 3-LIKE1; FBP: FITBINDING PROTEIN; MED16 AND 25: MEDIATOR SUBUNIT 16 and 25; ZAT12: ZINC FINGER OF ARABIDOPSIS THALIANA 12.

Figure 4. bHLH TFs network that regulates iron homeostasis in rice. Question mark indicates the putative degradation mechanism for OsPRI1 and OsPRI2 via OsHRZ2. The colour code for the bHLH TFs refers to the clades described Figure 2. Non bHLH proteins are in grey. IBP1.1: IDEF1-BINDING PROTEINS 1.1. 


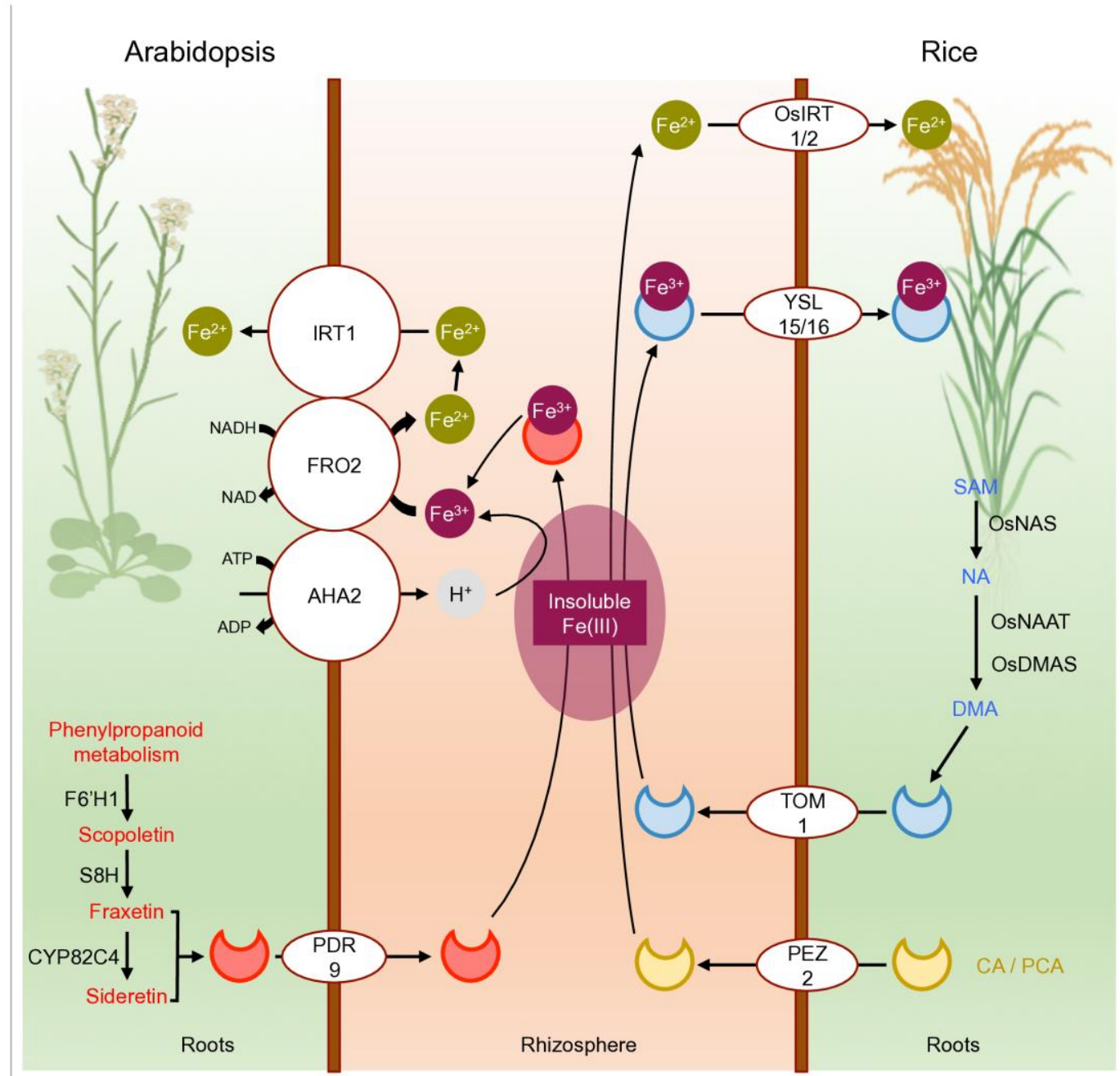

Figure 1. Schematic diagram of iron uptake strategies in Arabidopsis an rice. In Arabidopsis, AHA2 secretes protons into the rhizosphere to increase $\mathrm{Fe}^{3+}$ solubility; PDR9 secretes $\mathrm{Fe}$-mobilizing coumarins (i.e. fraxetin and sideretin) to mobilize and chelate $\mathrm{Fe}^{3+}$. $\mathrm{Fe}^{3+}$ is reduced into $\mathrm{Fe}^{2+}$ and is subsequently transported into the root epidermal cells by FRO2 and IRT1, respectively. AHA2, FRO2 and IRT1 form a protein complex optimizing $\mathrm{Fe}$ acquisition by creating a local environment with low $\mathrm{pH}$ and high $\mathrm{Fe}^{2+}$ concentration. Rice biosynthesizes and secrets DMA to chelate $\mathrm{Fe}^{3+}$. $\mathrm{Fe}^{3+}-\mathrm{DMA}$ complexes are transported into root cells via YSL15 and YSL16. In addition, rice also uptake $\mathrm{Fe}^{2+}$ from the soil through the activity of two $\mathrm{Fe}^{2+}$ transporters, OsIRT1 and OsIRT2 under waterlogged soil condition. The secretion of CA and PCA via the PEZ2 phenolics efflux transporter participates to $\mathrm{Fe}^{3+}$ mobilization and reduction into $\mathrm{Fe}^{2+}$. AHA2: $\mathrm{H}^{+}$-ATPase 2; PDR9: PLEIOTROPIC DRUG RESISTANCE 9/ATP-BINDING CASSETTE G37; FRO2: FERRIC REDUCTION OXIDASE 2; IRT1: IRON-REGULATED TRANSPORTER 1, F6'H1: FERULOYL CoA 6' HYDROXYLASE 1; S8H: SCOPOLETIN 8-HYDROXYLASE; CYP82C4: FRAXETIN 5-HYDROXYLASE; TOM1: TRANSPORTER OF MUGINEIC ACID 1; YSL15/16: YELLOW STRIPE-LIKE 15/16; OsIRT1/2: RICE IRONREGULATED TRANSPORTER 1/2; PEZ2: PHENOLICS EFFLUX ZERO 2; OsNAS: NICOTIANAMINE SYNTHASE; OsNAAT: NICOTIANAMINE AMINOTRANSFERASE; OsDMAS: DEOXYMUGINEIC ACID SYNTHASE; SAM: Sadenosyl methionine; NA: nicotianamine; CA: caffeic acid; PCA: protocatechuic acid. 
bHLH

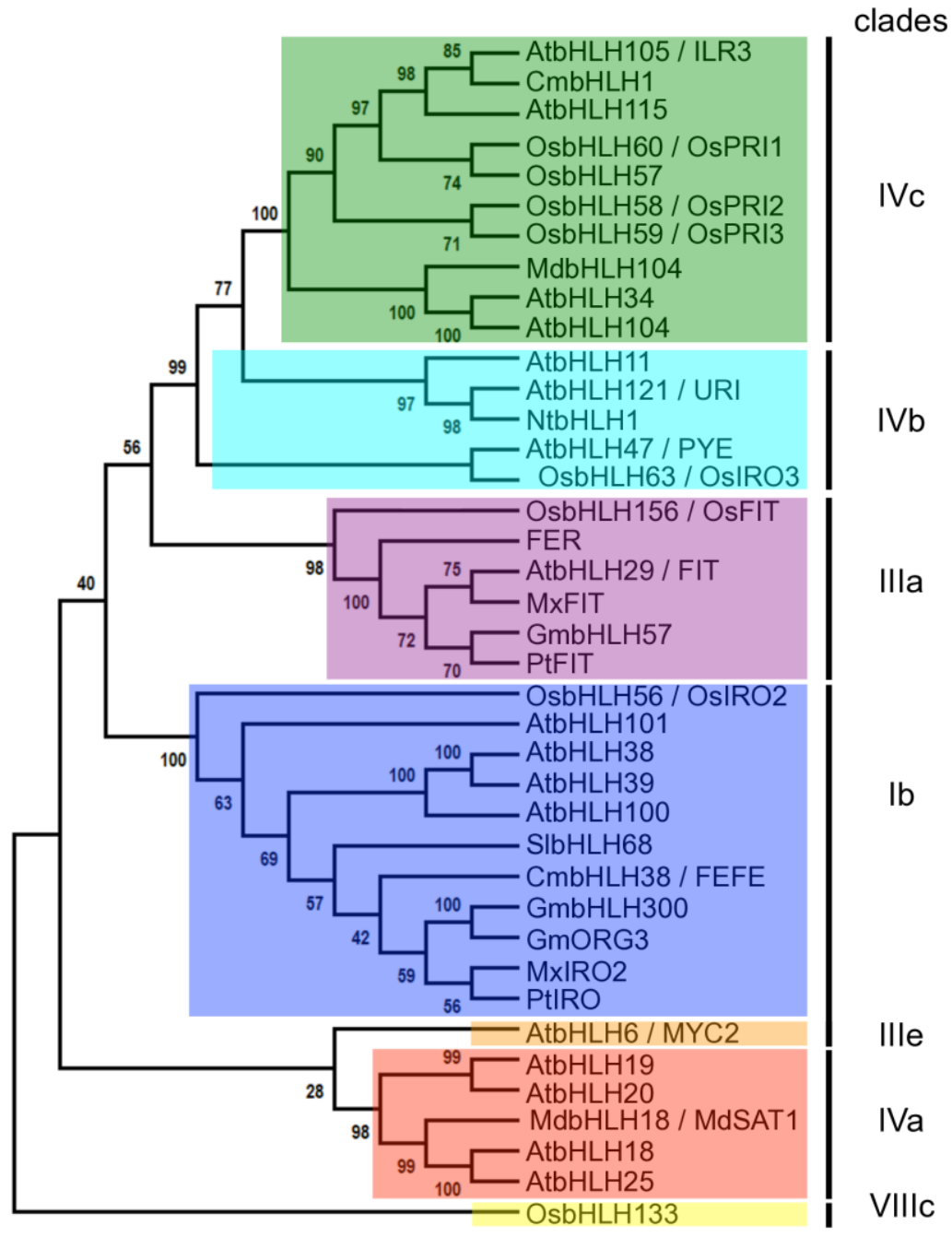

Figure 2. Phylogenetic tree of bHLH TFs involved in the regulation of iron homeostasis in plants. The tree was constructed by using the neighbor-joining method with the MEGA software (v10.0.5) using full-length bHLH amino acid sequences. The bootstrap analysis was carried out with 1000 replicates. Sequences were aligned, prior to the construction of the phylogenetic tree, using the ClustalX software (v2.0.11). The different bHLH clades are designated as previously reported (Heim et al., 2003). Species abbreviations: At, Arabidopsis thaliana; Md, Malus domestica; $\mathrm{Nt}$, Nicotiana tabacum; Os, Oryza sativa; SI, Solanum lycopersicum; Gm, Glycine max; Mx, Malus xiaojinensis; Cm, (CmbHLH1), Chrysanthemum morifolium; Cm (CmbHLH38), Cucumis melo; Pt, Populus tremula. 


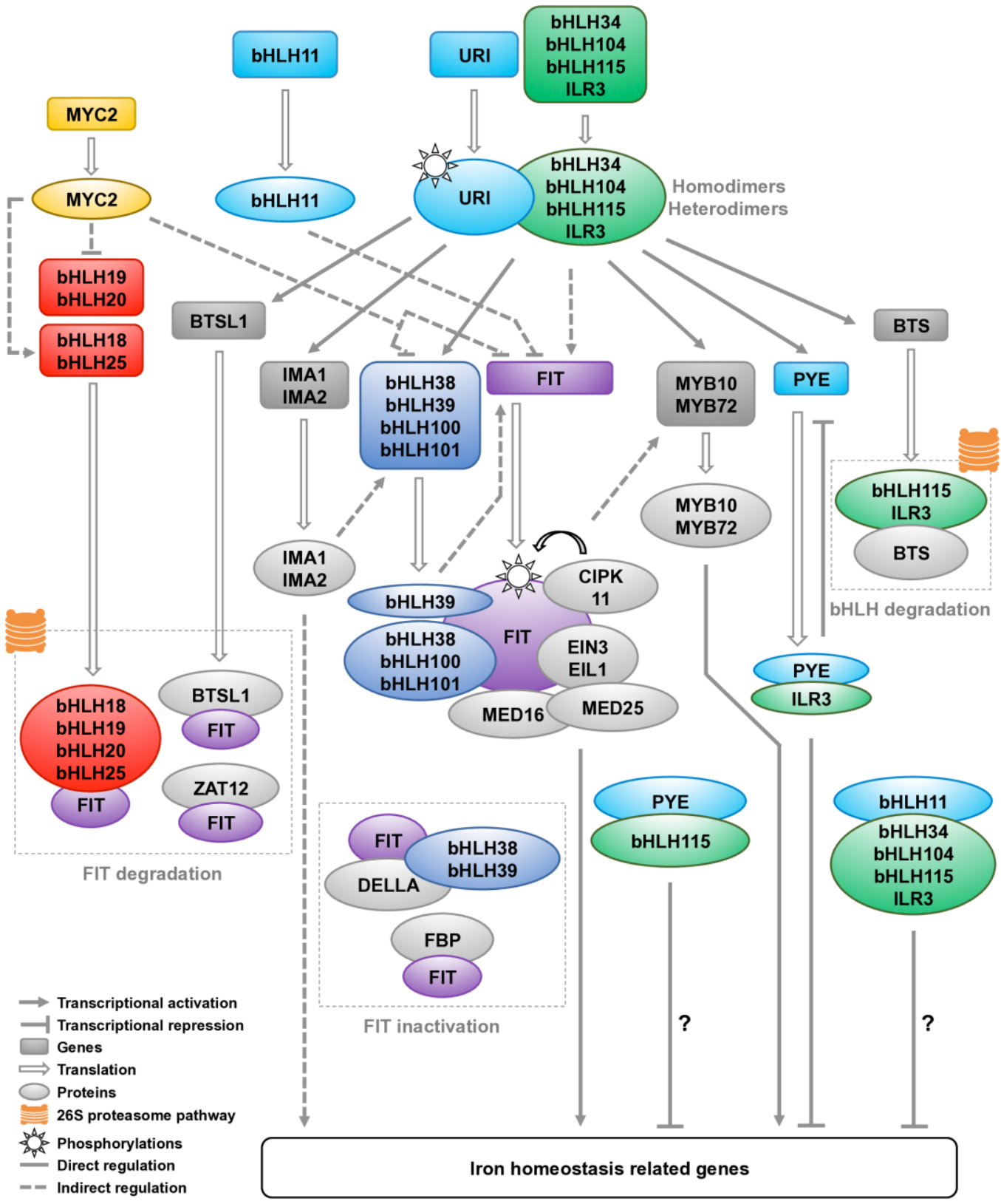

Figure 3. bHLH TFs network that regulates iron homeostasis in Arabidopsis. Question marks indicate protein complexes for which transcriptional activities have not yet been clearly demonstrated. The color code for the bHLH TFs refers to the clades described Figure 2. EIN3 and EIL1 favor FIT stability whereas MED16 and MED25 mediate the FIT/bHLH29-dependent iron deficiency responses (for additional details on posttranslational regulation of FIT see Kobayashi, 2019; Schwarz and Bauer, 2020; Spielmann and Vert, 2020; Wu and Ling, 2019). Non bHLH proteins are in grey. EIN3: ETHYLENE INSENSITIVE 3; EIL1: ETHYLENE INSENSITIVE 3-LIKE1; MED16 and 25: FBP: FITBINDING PROTEIN; MED16 AND 25: MEDIATOR SUBUNIT 16 and 25; ZAT12: ZINC FINGER OF ARABIDOPSIS THALIANA 12. 


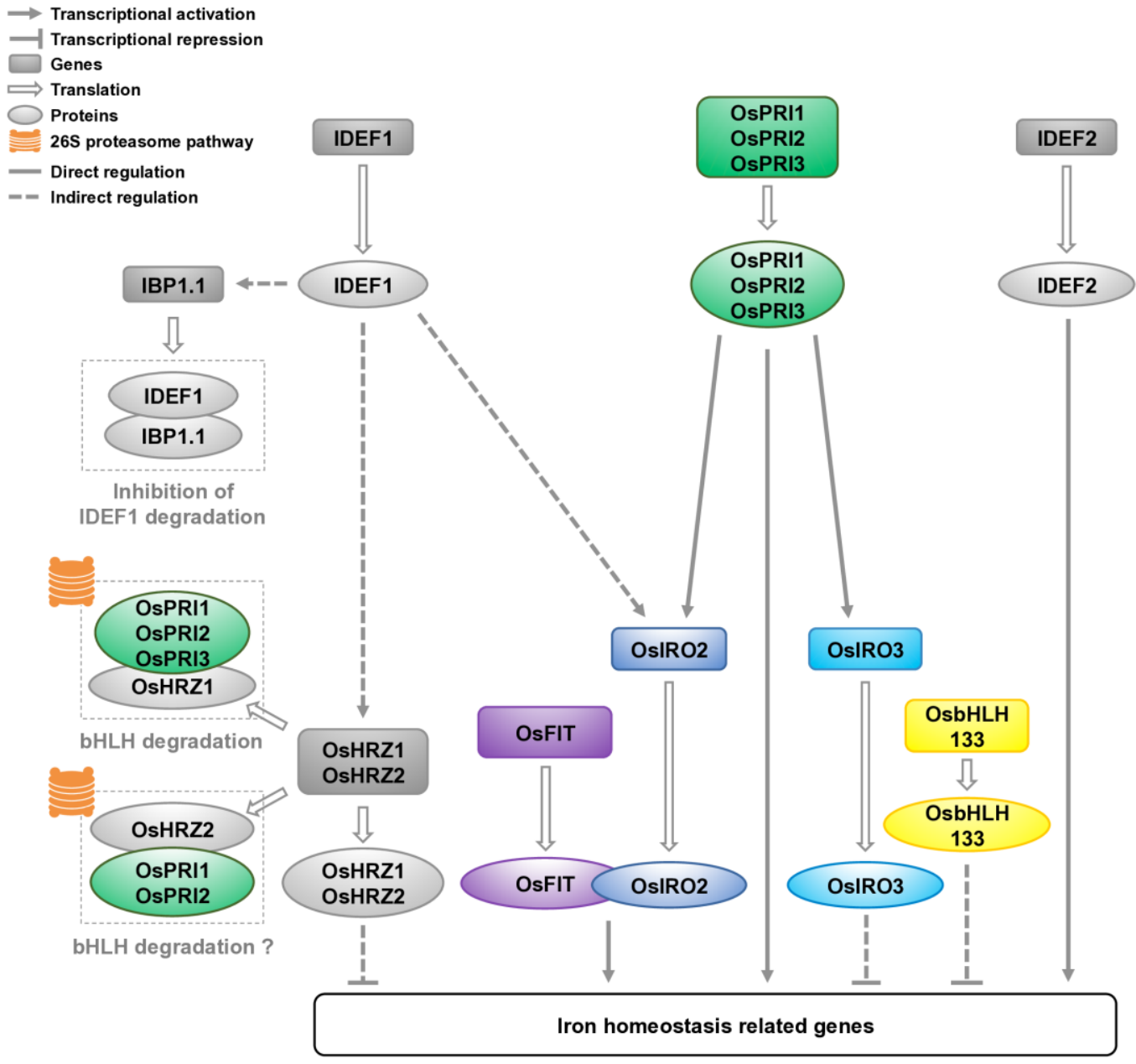

Figure 4. bHLH TFs network that regulates iron homeostasis in rice. Question mark indicates the putative degradation mechanism for OsPRI1 and OsPRI2 via OsHRZ2. The color code for the bHLH TFs refers to the clades described Figure 2. Non bHLH proteins are in grey. IBP1.1: IDEF1-BINDING PROTEINS 1.1. 